\title{
Self-assembled supramolecular hydrogels based on polymer-cyclodextrin inclusion complexes for drug delivery
}

\author{
Jun Li* \\ National University of Singapore, Singapore
}

The supramolecular self-assembly of cyclodextrins (CDs) and polymers has led to the development of novel supramolecular hydrogels for drug delivery applications. Many different supramolecular hydrogels have been formed between CDs and poly(ethylene oxide) or its copolymers, and the reversible thixotropism of these hydrogels makes them particularly suitable for delivery by syringe injection. For the long-term controlled release of drugs, supramolecular hydrogels with better controlled-release properties can be realized by assembling $\alpha-C D$ with various triblock copolymers bearing a hydrophobic block. The intermolecular interactions induced by the presence of hydrophobic blocks strengthen the molecular network and stabilizes the resultant hydrogel. The development of supramolecular hydrogels based on inclusion complexation between CDs and polymers opens up a new approach for designing novel drug delivery systems.

A supramolecular system is composed of two or more molecular entities held together and organized by means of intermolecular non-covalent binding interactions [1]. Supramolecular structures involving macrocyclic compounds have attracted tremendous interest not only as models for understanding natural supramolecular self-assembly and molecular recognition, but also as precursors for designing novel nanomaterials for electronics, biomedical and pharmaceutical applications [2-11]. Cyclodextrins (CDs), a series of natural cyclic oligosaccharides composed of 6,7 or $8 \mathrm{D}(+)$-glucose units linked by $\mathrm{D}(+)-1,4$-linkages (termed $\alpha-, \beta$ - and $\gamma$-CD, respectively), are well-suited for use in supramolecular systems. The geometry of CDs produces a hydrophobic inner cavity with a depth of ca. $7.0 \AA$ and an internal diameter of ca. $4.5,7.0$ or $8.5 \AA$ ( $\alpha$-, $\beta$ - and $\gamma$-CD, respectively). Various molecules can be inserted into the cavities of CDs to form supramolecular inclusion complexes, and such systems have been studied extensively as models for understanding the mechanism of molecular recognition [12-16].

A three-dimensional crosslinked network formed from a hydrophilic polymer, referred to as a hydrogel, can retain large volumes of water, causing the network to swell in solution. The extent of swelling and the content of water retained are largely dependent on the hydrophilicity of the polymer chains and the crosslinking density. The crosslinks can be formed by either covalent bonds or physical cohesion forces between the polymer segments, such as ionic bonds, hydrogen bonds, van der Waals forces and hydrophobic interactions [17-20]. Polymeric hydrogels are of great interest for biomaterials applications because of their biocompatibility $[21,22]$, and due to their high water content, they are attractive for the delivery of delicate bioactive agents such as proteins $[18,23,24]$. For example, chemically crosslinked poly(ethylene oxide) (PEO) hydrogels have been studied extensively for this purpose [25-27]. However, requiring covalent crosslinking for gelation, many hydrogels can only be applied as implantables, and the incorporation of drugs by sorption may be time-consuming and

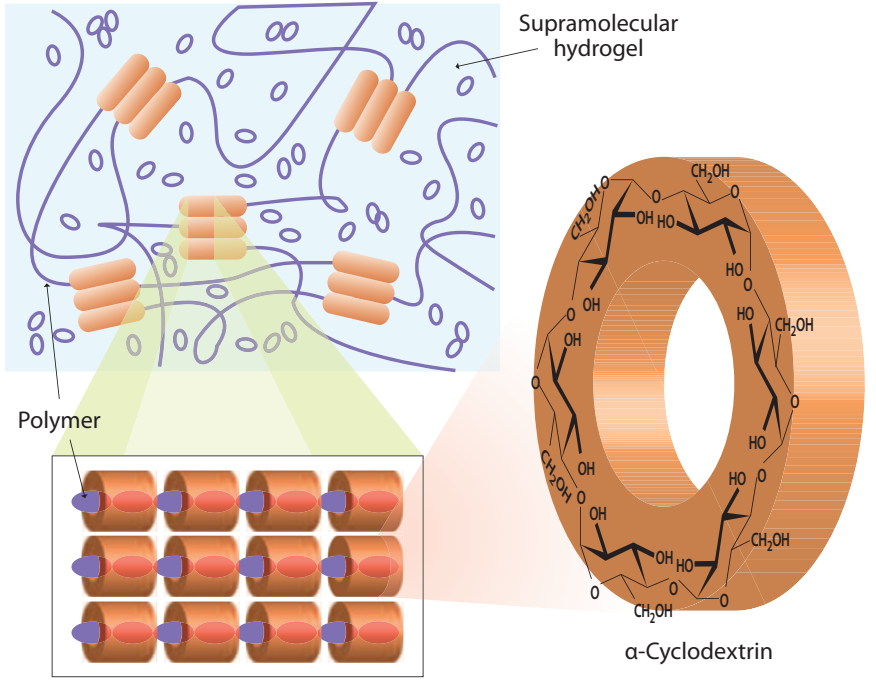

Figure 1. Formation of a supramolecular hydrogel of a-CD and high-molecularweight PEO by physical crosslinking through the self-assembly of inclusion complex domains. Adapted from Ref. 37, reproduced with permission (๔ 1994 Society of Polymer Science, Japan).

provide only limited loading potential. For drug delivery, the crosslinking reaction may also conjugate the drug to the hydrogel or impair the drug's chemical integrity, while the hydrogel itself may become nonbiodegradable with an ill-defined composition. A delivery formulation in which gelation and drug loading can be achieved simultaneously in an aqueous environment and without covalent crosslinking is therefore an attractive prospect. 

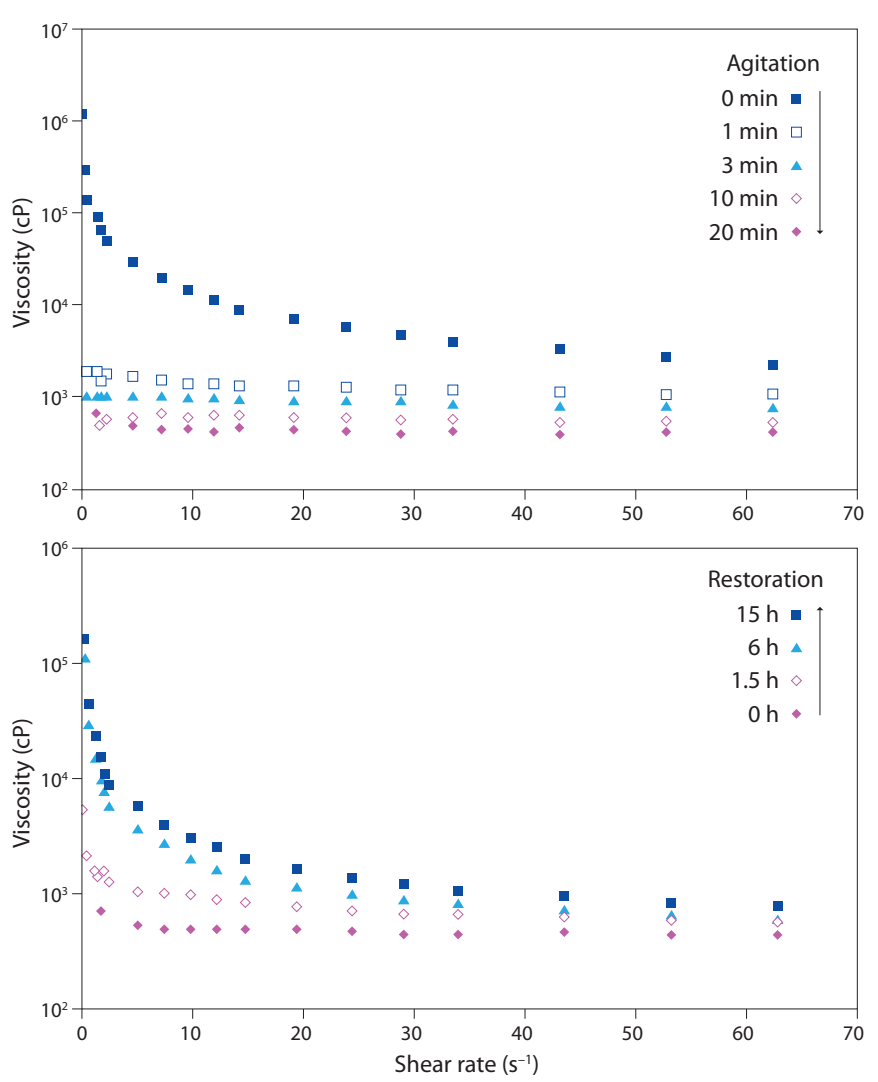

Figure 2. Change in the viscosity of hydrogels of a-CD and 20,000 Da PEO. (Upper) Change in viscosity with agitation at a shear rate of $120 \mathrm{~s}^{-1}$. (Lower) Restoration of original gel upon standing. Hydrogel composition: $43.5 \mathrm{mg}$ a-CD, $60 \mathrm{mg}$ PEO per $0.45 \mathrm{~mL}$. Modified after Ref. 38, reproduced with permission (@ 2003 Wiley-VCH Verlag $\mathrm{GmbH} \&$ (o. KGaA).

Physical hydrogels formed from synthetic copolymers and their potential applications for drug delivery systems have attracted much attention [28-34]. As a typical example, the sol-gel transition behavior of triblock copolymers consisting of $\mathrm{PEO}$ and a central poly(propylene oxide) (PPO) have been studied extensively and utilized for the delivery of drugs such as polypeptides and proteins [28,29]. A number of novel physical hydrogel systems formed with temperature- or $\mathrm{pH}$-sensitive copolymers or based on complexation of enantiomeric polymer or polypeptide segments have also been reported [30-34].

Recently, hydrogels formed based on the polymer-cyclodextrin inclusion complexes have emerged as another series of promising physical hydrogels suitable for controlled drug delivery [35,36]. The introduction of a suitable hydrophilic polymer in the inclusion complex in CD-based supramolecular systems can induce the spontaneous formation of a three-dimensional physically crosslinked macromolecular network, or hydrogel. The first report of a CD inclusion polymer forming a hydrogel, dating back to 1994 , describes the discovery of a sol-gel transition during inclusion complex formation between $\alpha-C D$ and high-molecular-weight PEO in aqueous solution [37]. The gelation was induced by the partially formed a-CD/PEO inclusion complex, which self-assembled into waterinsoluble domains in a physical crosslinking-like manner (Figure 1). This review summarizes recent developments in the preparation of physical hydrogels based on supramolecular self-assembly induced by inclusion complexation between CDs and polymers or copolymers for controlled drug delivery applications. Although there have been many recent reports on chemical or physical hydrogels consisting of CDs, the present review focuses exclusively on those systems in which gelation is induced by complexation between CDs and polymer chains.

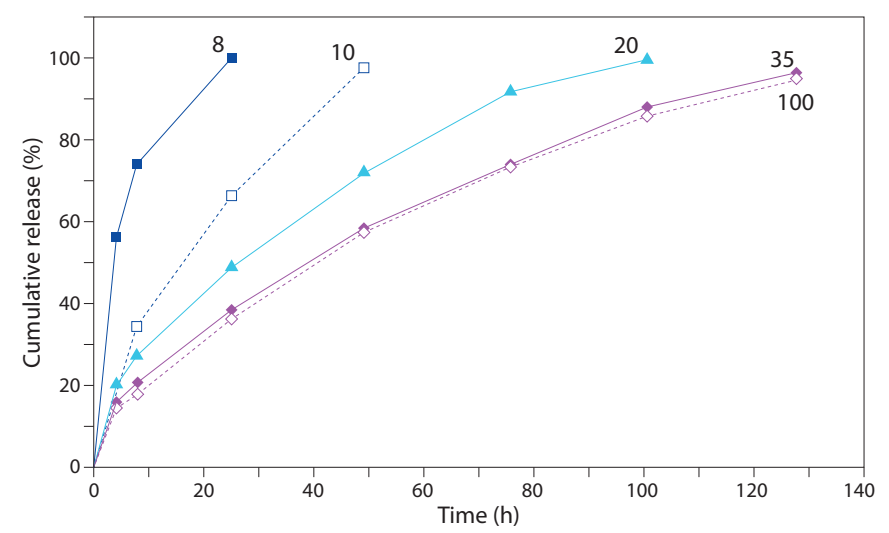

Figure 3. In vitro release profiles for dextran-FITC $(20,000 \mathrm{Da})$ from a-CD/PEO hydrogels formed with PEO of various molecular weights. Hydrogel composition: $3 \mathrm{mg}$ dextran-FITC, $43.5 \mathrm{mg}$ a-CD, $60 \mathrm{mg}$ PEO per $0.45 \mathrm{~mL}$. Labels denote average molecular weight of PEO $(\times 1,000 \mathrm{Da})$. Data from Ref. 38, reproduced with permission $(\odot 2003$ Wiley-VCH Verlag GmbH \& Co. KGaA).

\section{Injectable drug delivery systems based on $a-C D / P E O$ supramolecular hydrogels}

Linear water-soluble polymers such as PEO can penetrate the inner cavity of $a-C D$ to form inclusion complexes with a necklace-like supramolecular structure. The inclusion complexes of $a-C D$ and PEO precipitate from aqueous solution, indicating that the resulting complexes self-assemble into larger supramolecular structures with altered hydrophilicity. The first example of hydrogel formation between CDs and polymers was demonstrated through the supramolecular self-assembly of part of the chain of high-molecularweight $\mathrm{PEO}$, which resulted in a sol-gel transition of the aqueous a-CD/PEO solution [37].

As both CDs and PEO are known to be biocompatible and bioabsorbable, a new class of injectable drug delivery systems based on supramolecular hydrogels of these components has been developed [38]. These supramolecular hydrogels are reversibly thixotropic; the viscosity of the hydrogel decreases markedly when agitated, and is eventually restored, within hours in most cases, in the absence of agitation (Figure 2). The thixotropic property allows formulations of this hydrogel to be injected using a fine needle as a unique injectable hydrogel drug delivery system. Bioactive agents such as drugs, proteins, vaccines or plasmid DNAs could be incorporated into the gel and loaded into a syringe at room temperature without contact with organic solvents. The drug-bearing hydrogel formulation could then be injected into tissue under pressure as a reservoir for controlled drug release. Compared to implantable hydrogels, such an injectable hydrogel is particularly attractive. Investigations of the effect of syringe injection on the viscosity of the hydrogels have revealed that the viscosity dropped significantly during injection [38].

The drug-delivery properties of supramolecular hydrogels have also been evaluated in vitro using a model system of fluorescein isothiocyanate-labeled dextran (dextran-FITC) [38]. Figure 3 shows the in vitro release profiles of dextran-FITC from hydrogels with PEOs of various molecular weights. The release rate decreases sharply with increasing molecular weight of the PEO up to $35,000 \mathrm{Da}$, presumably due to chain entanglement and variations in complex stability. The release rate is relatively steady over time for gels formed with $35,000 \mathrm{Da}$ and $100,000 \mathrm{Da}$ PEO. Although gels formed from $\alpha-\mathrm{CD}$ and 100,000 Da PEO show the most sustained release kinetics, the data are primarily only of use for understanding the structure-property relationship because $\mathrm{PEO}$ of this size is undesirable for in vivo applications due to the difficulty of clearance from the body [38]. 


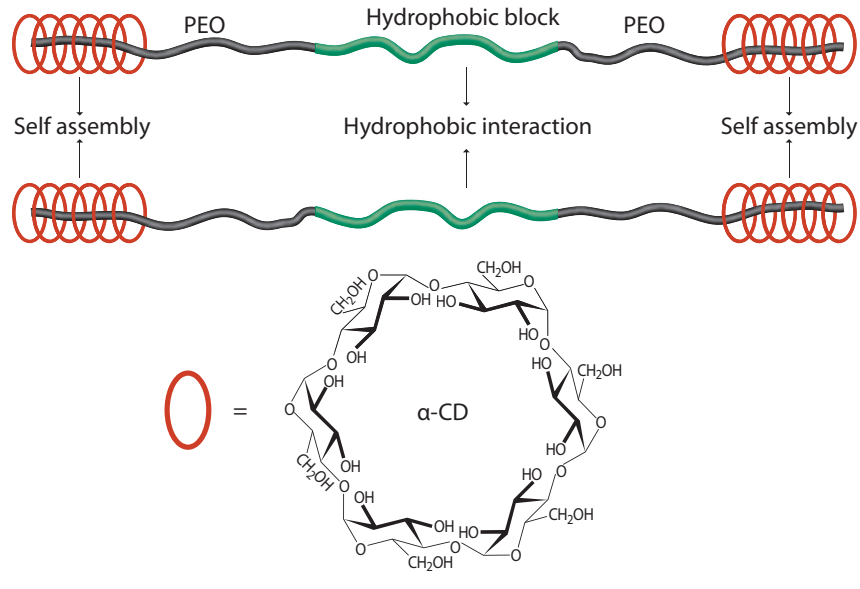

Figure 4. Schematic illustration of the supramolecular self-assembly of $a-C D$ and a triblock copolymer consisting of two PEO blocks flanking a central hydrophobic block. Adapted from Ref. 39 (๑ 2006 Elsevier).

\section{Development of a-cyclodextrin-based supramolecular hydrogels using block copolymers}

Triblock copolymers of poly(ethylene oxide) and poly(propylene oxide)

There are two main challenges in applying $\alpha-\mathrm{CD} / \mathrm{PEO}$ hydrogels for drug delivery: the typically fast release kinetics, which make hydrogels suitable only for short-term drug release over periods of less than one week; and the high molecular weight of the PEO (>10,000 Da), which makes the hydrogels unsuitable for application in vivo. The fast release kinetics of the hydrogels is determined by the rapid dissociation of the hydrogel in aqueous environments, which in turn is related to hydrophilic nature of PEO. Furthermore, as PEO is not biodegradable, this component of the hydrogel cannot be efficiently filtered out of the body by the kidneys due to large hydrodynamic radius of the high-molecular-weight PEO required for the formation of stable hydrogels. To develop supramolecular hydrogels with good, long-term controlled-release properties, our group proposed an alternative design for supramolecular hydrogels based on $\alpha-C D$ and various triblock copolymers in which PEO blocks flank a hydrophobic middle block [39]. The hypothesis behind this design is that the additional intermolecular hydrophobic interaction of the middle blocks can further strengthen the hydrogel network, resulting in more stable hydrogels for the long-term controlled release of drugs (Figure 4).

This idea was first proved using a supramolecular hydrogel system of $\alpha-C D$ and a Pluronic PEO-PPO-PEO triblock copolymer [40]. Aqueous solutions of PEO-PPO-PEO are known to form thermosensitive hydrogels at high concentrations [29,41]. The addition of a-CD, however, allows the formation of hydrogels of PEO-PPO$\mathrm{PEO}$ at much lower copolymer concentrations through the action of $\alpha-C D$ to form inclusion complexes with parts of the flanking PEO blocks [42].

The effect of $\alpha-C D$ in aiding the gelation of aqueous solutions of PEO-PPO-PEO copolymers was evidenced by phase diagrams of the aqueous mixtures of PEO-PPO-PEO and $\alpha-\mathrm{CD}$. In the temperaturepolymer concentration phase diagrams of aqueous PEO-PPO-PEO $\left(\mathrm{EO}_{13} \mathrm{PO}_{30} \mathrm{EO}_{13}\right)$ in the absence and presence of $\alpha-\mathrm{CD}$ at various concentrations, the region in which pure $\mathrm{EO}_{13} \mathrm{PO}_{30} \mathrm{EO}_{13}$ can form a hydrogel is very small and restricted to very high copolymer concentrations. With the addition of $\alpha-C D$, however, the gelation region expands with $\mathrm{a}-\mathrm{CD}$ concentration, and the lowest copolymer concentration at which the hydrogel can form becomes much lower. For example, at $31^{\circ} \mathrm{C}$, the lowest copolymer concentration that still allows gelation can be reduced from $50 \%$ to about $20 \%$ by the addition of
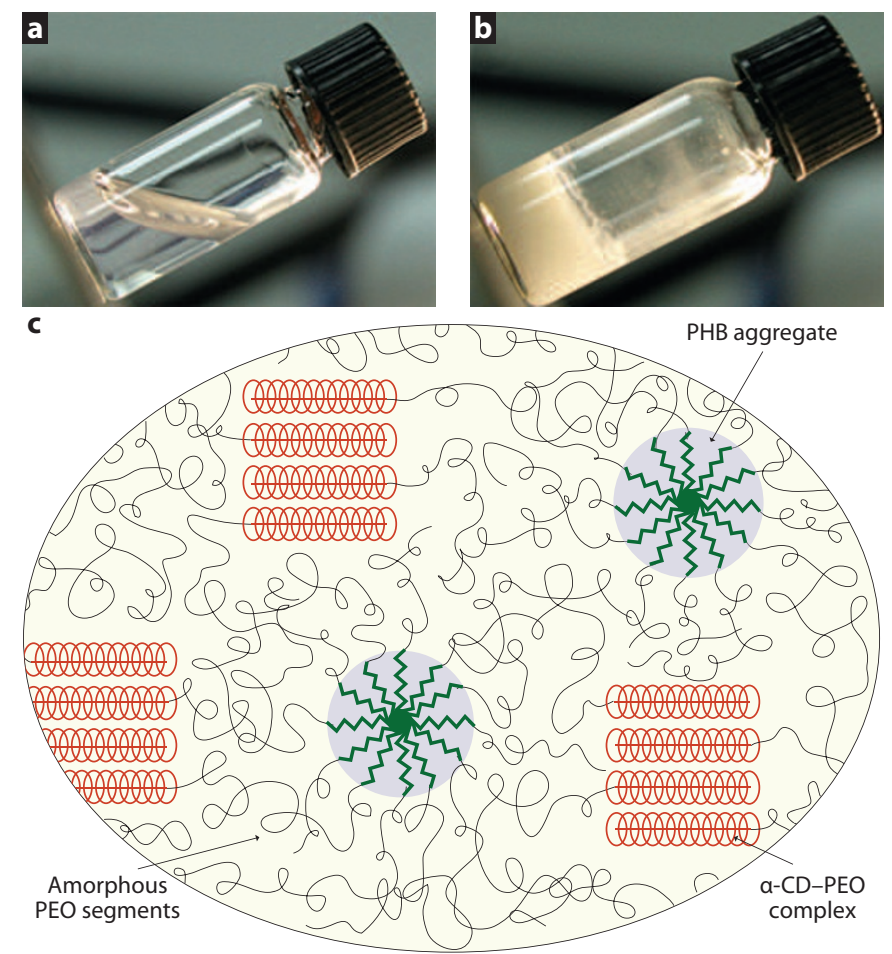

Figure 5. Gelation of PEO-PHB-PEO with $a-C D$. (a) Aqueous solution of PEO-PHB-PEO (5,000-1,750-5,000) triblock copolymer. (b) Supramolecular hydrogel of $a-C D / P E O-P H B-P E O(5,000-17,50-5,000)$. (c) Schematic illustration of the structure of the a-CD/PEO-PHB-PEO hydrogel. Adapted from Ref. 39 (๔ 2006 Elsevier).

$\alpha-C D$. It is clear that the formation of inclusion complexes between $\mathrm{EO}$ blocks of $\mathrm{EO}_{13} \mathrm{PO}_{30} \mathrm{EO}_{13}$ and $\alpha$-CD has a pronounced effect on the hydrophobicity of the PEO-PPO-PEO copolymer, resulting in a significant lowering of the gelation concentration [42].

The in vitro controlled release properties of the a-CD/PEO-PPOPEO hydrogel have been studied using FITC-labeled bovine serum albumin (BSA) as a model protein drug (X. P. Ni, J. Li, unpublished data). In that study, hydrogels with triblock copolymers of various molecular weights and chain compositions were prepared and found to display a wide range of release kinetics. Hydrogels with $\mathrm{EO}_{10} \mathrm{PO}_{44} \mathrm{EO}_{10}$ sustained the release of BSA for more than one week with linear release kinetics, whereas as other formulations, particularly with $\mathrm{EO}_{113} \mathrm{PO}_{57} \mathrm{EO}_{113}, \mathrm{EO}_{13} \mathrm{PO}_{30} \mathrm{EO}_{13}, \mathrm{EO}_{11} \mathrm{PO}_{16} \mathrm{EO}_{11}$ and $\mathrm{EO}_{76} \mathrm{PO}_{30} \mathrm{EO}_{76}$, appeared to be too unstable for long-term sustained delivery. The optimal composition of PEO-PPO-PEO for protein delivery in these supramolecular hydrogels is $25-30 \mathrm{wt} \%$ PEO. A good balance between the PEO and PPO block lengths is therefore very important. Recently, there has been a report on the formation of a supramolecular hydrogel between $a-C D$ and PEO-PPO-PEO hybridized with carbon nanotubes (CNTs) [43]. The CNTs were hybridized into the supramolecular hydrogel to reduce the network density and hence improve the diffusion of encapsulated substances. The modulus of the hydrogels was found to rise slightly after introducing CNTs. The antimicrobial activity, skin-adhesion and water-retention properties of the supramolecular hybrid hydrogels were also studied for wounddressing applications.

Triblock copolymers of poly(ethylene oxide) and poly $((\mathrm{R})-3$-hydroxybutyrate)

In addition to PEO-PPO-PEO triblock copolymers, our group has synthesized investigated the formation of hydrogels using a block copolymer in which the middle block is replaced with poly $((\mathrm{R})$ 3-hydroxybutyrate) (PHB) [44]. PHB is a natural biopolyester, and is biodegradable and potentially biocompatible, making it a promising 
candidate for biomedical applications. The PEO-PHB-PEO triblock copolymer with short PEO blocks $(2,000 \mathrm{Da})$ was found to form crystalline inclusion complexes with a-CD $[45,46]$, whereas hydrogels could be formed with PEO-PHB-PEO copolymers with longer PEO blocks (5,000 Da) (Table 1) [39]. Although the two copolymers were water-soluble, they formed micelles in aqueous solution at very low concentrations. The critical micelle concentration of PEO-PHB-PEO was found to be significantly lower than that for Pluronic PEO-PPO$\mathrm{PEO}$ or a PEO-based triblock copolymer bearing a central polylactic acid block at similar compositions [47]. The results indicate that the PHB block in PEO-PHB-PEO has a very strong tendency towards self-assembly in aqueous environments.

Aqueous solutions of PEO-PHB-PEO formed micelles but not hydrogels at a reference copolymer concentration of $13 \mathrm{wt} \%$ [39]. On the addition of $a-C D$, however, hydrogels could be obtained at minimum concentrations of $13 \mathrm{wt} \%$ PEO-PHB-PEO and $9.7 \%$ $a-C D$ at room temperature (Figure 5). These results demonstrate that a-CD aids gel formation and allows hydrogels of PEO-PHB-PEO to be formed at relatively low copolymer concentrations. The inclusion complexes formed by $\alpha-C D$ and PEO blocks are considered to be pivotal in promoting hydrogel formation by aggregating into microcrystals, which act as physical crosslinks inducing the formation of a supramolecular polymer network. Micellization of the PHB block is also important in the gelation process; hydrophobic interactions between PHB blocks facilitate formation of a polymer network. The gelation of aqueous solutions of PEO-PHB-PEO triblock copolymers and $\alpha-C D$ is therefore considered to be the result of the cooperative action of inclusion complexation between $\alpha-C D$ and PEO blocks and micellization of the PHB block of the triblock copolymer.

$\mathrm{X}$-ray diffraction studies have provided data on the possible structure of a-CD/PEO-PHB-PEO supramolecular hydrogels

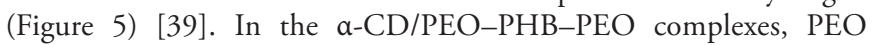
chains penetrate $\alpha-C D$ rings from both ends to form necklace-like inclusion complexes. The molecular weight of each PEO block is ca. 5,000 Da in these supramolecular hydrogels. However, the length of PEO from each end of the copolymer that could be included in a-CD rings is equivalent to a molecular weight of about $2,000 \mathrm{Da}$ based on studies of complex formation in these system, which found that only crystalline solid complexes could be obtained at PEO lengths of $2,000 \mathrm{Da}$ or less $[45,46]$. This hypothesis is supported by the number of $a-C D$ rings that can be threaded onto a PEO chain and capped by bulky groups at the two ends, as described in a study on the synthesis of $\mathrm{a}-\mathrm{CD}-\mathrm{PEO}$ polyrotaxanes [48]. In the course of preparing polyrotaxanes in that study, a maximum average number of 23 a-CD rings, corresponding to a length of $\mathrm{PEO}$ equivalent to a molecular weight of 2,000 Da, were trapped over each PEO chain, irrespective of whether longer PEO blocks were used. In the case of PEO-PHB-PEO block copolymers with a PEO block length of $5,000 \mathrm{Da}$, the $\mathrm{PEO}$ segments covered by $\mathrm{a}-\mathrm{CD}$ rings can therefore be

Table 1. PEO-PHB-PEO triblock copolymers tested for hydrogel formation with a-CD

\begin{tabular}{|c|c|c|c|c|c|}
\hline \multirow[b]{2}{*}{ Triblock copolymer ${ }^{a}$} & \multirow[b]{2}{*}{$M_{\mathbf{n}}^{b}$} & \multirow[b]{2}{*}{$M_{\mathbf{w}}^{b}$} & \multirow[b]{2}{*}{$M_{\mathrm{w}} / M_{\mathrm{n}}^{b}$} & \multicolumn{2}{|c|}{ Block length $\left(M_{n}\right)$} \\
\hline & & & & PEO $^{b}$ & $\mathbf{P H B}^{c}$ \\
\hline $\begin{array}{l}\text { PEO-PHB-PEO } \\
(5,000-1,750-5,000)\end{array}$ & 11,270 & 15,250 & 1.35 & 4,830 & 1,750 \\
\hline $\begin{array}{l}\text { PEO-PHB-PEO } \\
(5, \mathrm{~S} 000-3,140-5,000)\end{array}$ & 12,160 & 16,430 & 1.35 & 4,830 & 3,140 \\
\hline \multicolumn{6}{|c|}{ a Numbers in parentheses denote indicative block length in $\mathrm{g} / \mathrm{mol}$. } \\
\hline \multicolumn{6}{|c|}{$\begin{array}{l}{ }^{b} \text { Number-average molecular weight }\left(M_{n}\right) \text {, weight-average molecular weight }\left(M_{w}\right) \text {, } \\
\text { and molecular weight polydispersity }\left(M_{n} / M_{w}\right) \text { determined by gel permeation } \\
\text { chromatography. }\end{array}$} \\
\hline \multicolumn{6}{|c|}{$\begin{array}{l}\text { ' } \text { Block lengths determined by }{ }^{~} \mathrm{H} \text { nuclear magnetic resonance and gel permeation } \\
\text { chromatography. }\end{array}$} \\
\hline
\end{tabular}

expected to be shorter than 2,000 Da from each end. The cooperative gelation process in effect hinders further threading of $a-C D$ over the PEO blocks. The cooperative effect of partial inclusion complexation of $a-C D$ with PEO blocks and the hydrophobic interaction between the middle PHB blocks results in a self-assembling system that forms a strong network and a novel supramolecular hydrogel.

To examine the in vitro controlled-release properties of a-CD/PEO-PHB-PEO hydrogels, our group has studied this system using dextran-FITC as a model macromolecular drug [39]. Figure 6 shows the release profiles of various $\alpha-\mathrm{CD} / \mathrm{PEO}-\mathrm{PHB}-\mathrm{PEO}$ hydrogels encapsulating dextran-FITC as tested in the in vitro release kinetics studies. PEO-PHB-PEO alone at a concentration of $13 \mathrm{wt} \%$ in water could not sustain the release of dextran-FITC due to immediate dispersion in an excess of water or phosphate buffered saline (PBS). The a-CD/PEO homopolymer hydrogel dissociated and dissolved in PBS within five days even at very high PEO molecular weights $(35,000 \mathrm{Da})$. However, the $\mathrm{a}-\mathrm{CD} / \mathrm{PEO}-\mathrm{PHB}-\mathrm{PEO}(5,000-3,140-5,000)$ hydrogels with reasonably high $\alpha-C D$ concentration $(9.7 \mathrm{wt} \%)$ showed excellent controlled-release properties, sustaining the release of dextran-FITC for more than one month. The hydrogels with lower $\mathrm{a}-\mathrm{CD}$ concentrations displayed much faster release kinetics, indicating that the complexation between $\alpha-C D$ and the PEO blocks plays a key role in forming a stable supramolecular hydrogel. Interestingly, the $\mathrm{a}-\mathrm{CD} / \mathrm{PEO}-\mathrm{PHB}-\mathrm{PEO}$ $(5,000-1,750-5,000)$ hydrogel with shorter PHB blocks only sustained

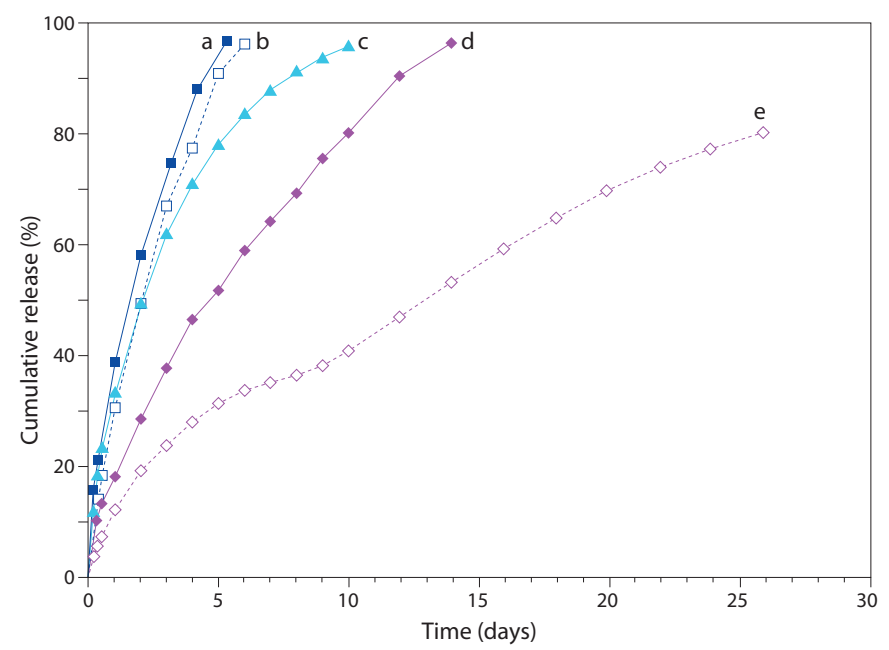

Figure 6. In vitro release profiles for dextran-FITC (20,000 Da) from a-CD/PEO hydrogel and a-CD/PEO-PHB-PEO hydrogels with various compositions. Labels a-e refer to hydrogels listed in Table 2 . Modified after Ref. 39, reproduced with permission (C) 2006 Elsevier).

Table 2. a-CD/PEO-PHB-PEO supramolecular hydrogel formulations used for in vitro controlled release studies

\begin{tabular}{|c|c|c|c|c|c|}
\hline \multirow[b]{3}{*}{ Hydrogel } & \multirow[b]{3}{*}{ Polymer } & \multicolumn{4}{|c|}{ Gel composition (wt\%) } \\
\hline & & \multirow[b]{2}{*}{$\alpha-C D$} & \multicolumn{3}{|c|}{ Dextran- } \\
\hline & & & Polymer & FITC & $\mathrm{H}_{2} \mathrm{O}$ \\
\hline $\mathrm{a}$ & PEO $(35,000)$ & 9.7 & 13.3 & 0.66 & 76.3 \\
\hline $\mathrm{b}$ & $\begin{array}{l}\text { PEO-PHB-PEO } \\
(5,000-1,750-5,000)\end{array}$ & 9.7 & 13.3 & 0.66 & 76.3 \\
\hline $\mathrm{c}$ & $\begin{array}{l}\text { PEO-PHB-PEO } \\
(5,000-3,140-5,000)\end{array}$ & 5.4 & 13.3 & 0.66 & 80.6 \\
\hline $\mathrm{d}$ & $\begin{array}{l}\text { PEO-PHB-PEO } \\
(5,000-3,140-5,000)\end{array}$ & 7.9 & 13.3 & 0.66 & 78.1 \\
\hline $\mathrm{e}$ & $\begin{array}{l}\text { PEO-PHB-PEO } \\
(5,000-3,140-5,000)\end{array}$ & 9.7 & 13.3 & 0.66 & 76.3 \\
\hline
\end{tabular}




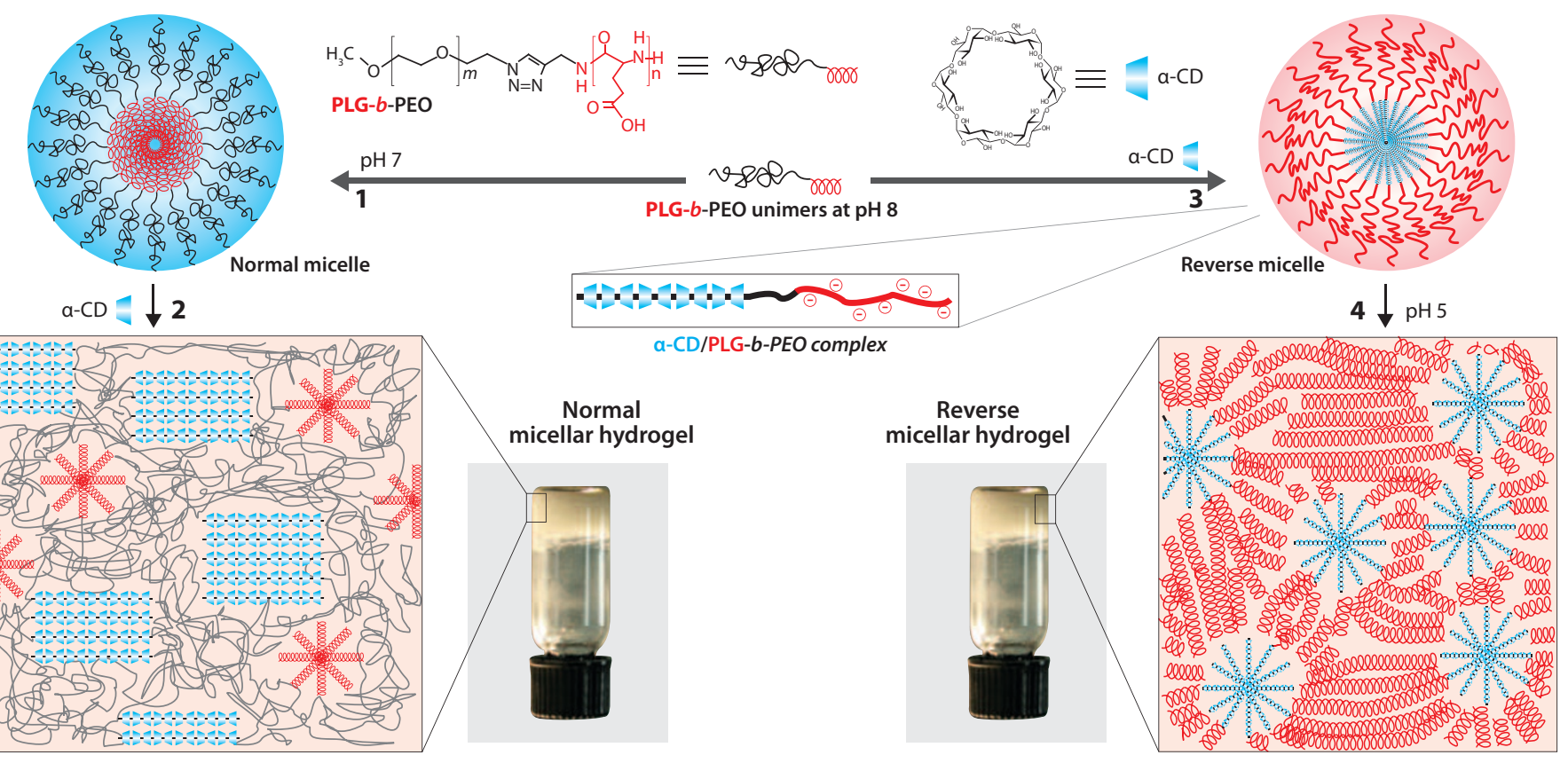

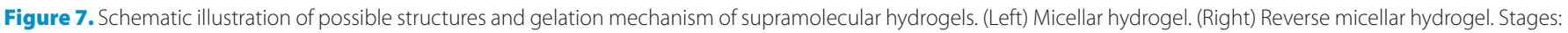

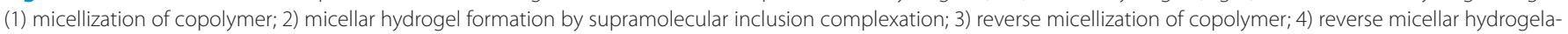
tion. Modified after Ref. 53, reproduced with permission (๑ 2010 Wiley-VCH Verlag GmbH \& Co. KGaA).

the release of dextran-FITC for six days, despite the high a-CD concentration $(9.7 \mathrm{wt} \%)$. These results indicate that the PHB block length is also a critical factor determining the stability of the supramolecular hydrogels, further supporting the hypothesis that the cooperative effect a-CD complexation with PEO segments and hydrophobic interaction between PHB blocks results in the formation of hydrogels with strong supramolecular networks and long-term sustained-release properties. Many simple triblock copolymer delivery systems have been unable to achieve such sustained-release properties. Our findings suggest that the properties of supramolecular hydrogels can be fine-tuned not only by adjusting the length of the PHB block, but also by using different copolymers, which could open up a wide range of possible biomedical applications. Although supramolecular hydrogels of $\alpha-C D$ and homo-PEO

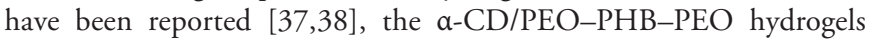
develop much stronger hydrogel networks by the cooperative effect of the self-assembly of inclusion complexes of $\alpha$-CD with PEO segments and the hydrophobic interaction between PHB segments. The result is a hydrogel capable of sustained release using lower-molecular-weight and biodegradable copolymers, which will have significant advantages with regard to bioabsorption after drug delivery and dissociation of the hydrogels into their components [39]. A recent report on enzymatic degradation of supramolecular complexes formed between $\alpha-C D$ and poly( $\varepsilon$-caprolactone) (PCL) [49] underlines good bioabsorbance of this type of supramolecular hydrogel.

It was found recently that $\mathrm{aPHB}-\mathrm{PEO}-\mathrm{aPHB}$ triblock copolymers (where $\mathrm{aPHB}$ denotes both enantiomers of $\mathrm{PHB}$ ) with low molecular weight $(<5,000 \mathrm{Da})$ formed unexpectedly strong hydrogels with a-CD, reaching storage modulus $\left(G^{\prime}\right)$ values of up to $10^{4}-10^{5} \mathrm{~Pa}$ [50]. The aPHB-PEO-aPHB triblock copolymers were synthesized so as to ensure the formation of well-defined polymer architectures that favor the formation of block-selected polypseudorotaxanes, with a-CD molecules preferentially covering the middle PEO block $[51,52]$. At sufficiently low a-CD coverage over the PEO segment, the polypseudorotaxanes obtained formed hydrogels instead of precipitates. Although this could be easily understood as resulting from an increase in hydrophilicity with greater PEO exposure on the polypseudorotaxane, the strength of the hydrogels prepared in this way was uncharacteristic of that for a guest polymer with a molecular weight of around 5,000 Da. It was concluded that the enhancement in gel formation was attributable to the hydrophobic association of the telechelic aPHB segments [50].

\section{Block copolymers of poly(ethylene oxide) and poly(L-glutamic acid)}

A diblock copolymer of poly(L-glutamic acid) (PLG) and PEO was recently reported to form a supramolecular hydrogel with $\alpha-C D$, and further to form reverse micellar hydrogels from the same complex at different $\mathrm{pH}$ via the cooperation combination of host-guest chemistry and hydrogen bonding interactions (Figure 7) [53]. These supramolecular hydrogels form as a result of hydrogen bonding interactions among PLG and supramolecular inclusion complexation between PEO and $a-C D$. The hierarchical mechanism giving the micellar hydrogels occurs through the formation of polypeptide-cored micelles with a PEO corona via hydrogen bonding-mediated self-assembly, followed by the physical crosslinking of micelles via the supramolecular inclusion complexation of PEO and $\alpha-\mathrm{CD}$. The reverse micellar hydrogel was produced through the formation of $\alpha-\mathrm{CD} / \mathrm{PEO}$ polypseudorotaxane-cored micelles with a polypeptide corona (reverse micelles) via self-assembly mediated by the supramolecular inclusion complexation, followed by the physical crosslinking of the reverse micelles via the hydrogen-bonding interactions among the polypeptide coronas. The reverse micellar hydrogel could be loaded to high concentrations $(10 \%)$ with the anticancer drug doxorubicin hydrochloride, with sustained release for up to 45 days. These properties make this system a potentially useful injectable drug delivery system for cancer therapy.

\section{Block copolymers of poly(ethylene oxide) and} poly( $\varepsilon$-caprolactone)

Other biopolyester-based block copolymers have also been used for supramolecular hydrogel formation and for drug delivery applications [54-56]. A diblock copolymer of PEO with PCL was used to form a supramolecular hydrogel with $\alpha-C D$, and the controlled-release properties of the hydrogel were studied using a model macromolecular drug [54]. Rheological studies indicate that the $\alpha$-CD/PEO-PCL hydrogel is reversibly thixotropic. Examination of the in vitro release kinetics of the $\alpha$-CD/PEO-PCL hydrogels using dextran-FITC revealed the PCL system to have much improved sustained release 

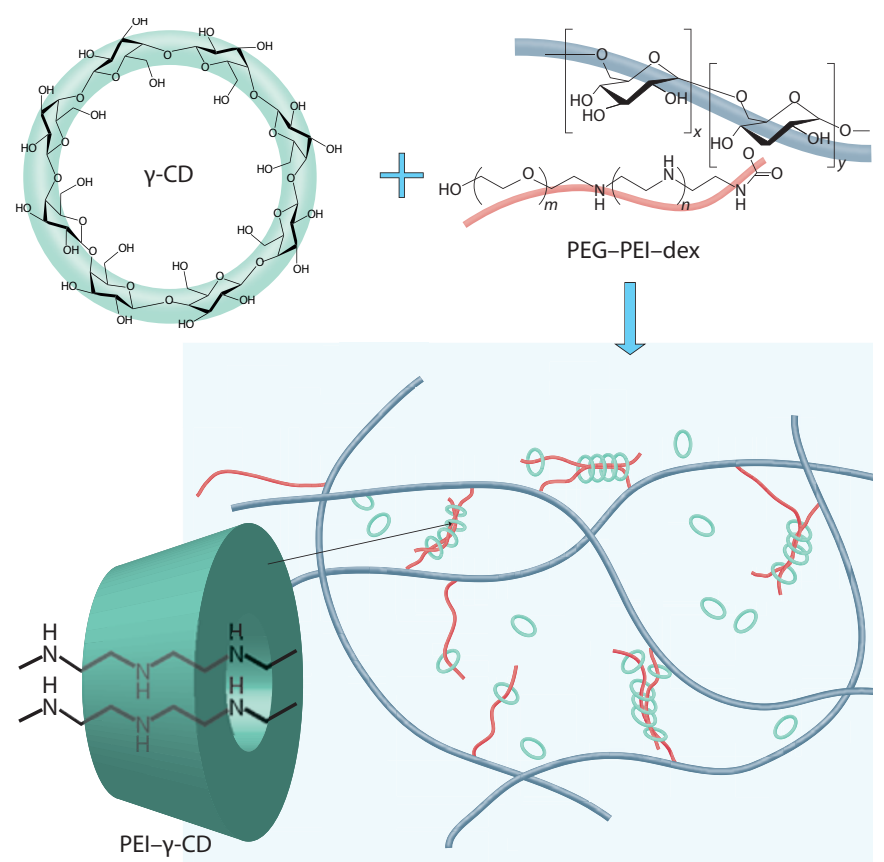

Figure 8. Schematic illustration of the $\gamma$-CD-mediated formation of hydrogels from dextrin, PEO and poly(ethyleneimine) (PEI). A supramolecular network is formed via double-strand complexation between $\gamma$-CD and the double strands of PEO-PEI chains to afford parallel or antiparallel complexes. a-CD can accommodate a single strand of PEl, whereas $\gamma$-CD can incorporate double strands. Modified after Ref. 61, reproduced with permission (๔ 2010 Wiley-VCH Verlag GmbH \& Co. KGaA).

properties compared with that of $\alpha$-CD/PEO supramolecular hydrogels, even with low diblock copolymer molecular weights. It was concluded that the incorporated hydrophobic PCL block reduces the molecular weight of PEO required for long-term drug release system. The sustained release properties were also found to be dependent on the $a-C D$ concentration in the supramolecular hydrogels.

The formation of supramolecular hydrogels from $\alpha-C D$ and PEO-PCL-PEO triblock copolymers has also been studied, and the resultant hydrogels have been used for the localized delivery of erythropoietin (EPO) in vitro and in vivo $[55,56]$. EPO can protect heart muscle tissue from ischemia-related damage, but it is also implicated in polycythaemia, which can cause thrombo-embolic complications. A therapy based on the highly localized sustained delivery of EPO directly to impaired tissue could therefore be effective. Local intramyocardial injection of EPO using a supramolecular hydrogel was investigated for its ability to enhance the cardioprotective effect without causing polycythaemia after myocardial infarction. Comparing the administration of PBS, an a-CD-PEO-PCL-PEO hydrogel, recombined human erythropoietin (rhEPO) in PBS, or rhEPO in hydrogel were injected into the infarcted area immediately after $\mathrm{MI}$ in rats. It was found that the hydrogel allowed a sustained release of EPO, which inhibited cell apoptosis and increased neovasculature formation, and subsequently reduced infarct size and improved cardiac function compared with other groups. Notably, there was no evidence of polycythaemia caused by the therapy with the supramolecular hydrogel delivery system, and there were no differences in erythrocyte count and hematocrit in the animals treated with hydrogel delivery system, PBS, or hydrogel blank injection. It was concluded that the intramyocardial delivery of rhEPO with aCD-PEO-PCL-PEO hydrogel may result in cardiac performance improvement after MI without apparent adverse effect [56].

\section{Poly(ethylene oxide)-grafted copolymers}

Following a physical crosslinking-like mechanism similar to the inclusion complexation between $a-C D$ and a segment of PEO in a block copolymer that leads to self-assembly of a hydrogel, Huh and co-workers reported the formation of supramolecular hydrogels using $\mathrm{CD}$ and several different PEO-grafted copolymers [57]. The supramolecular hydrogels were formed by inclusion complexation between $\alpha-C D$ and PEO-grafted dextrans in aqueous media. The sol-gel phase transition temperature of the system could be controlled by adjusting the polymer concentration and the PEO content in the graft copolymers, as well as by changing the stoichiometric ratio between the guest and host molecules. Similar hydrogels have been formed from $\alpha-C D$ and PEO-grafted chitosan [58], and $\alpha-C D$ and PEO-grafted hyaluronic acid [59]. A PPO-grafted dextran copolymer has also been used to form a thermoreversible hydrogel with $\beta$-CD by physical crosslinking induced by inclusion complexation between PPO segments and $\beta$-CD [60]. Recently, inclusion complexation between $\gamma$-CD and two PEO chains, where the PEO chain is grafted to a dextran, was used to form supramolecular polymer networks leading to gelation (Figure 8) [61,62]. In this case, the two PEO chains bundled within the $\gamma$-CD cavities promoted physical crosslinking for gelation.

Some of these hydrogels have been explored for controlled drug delivery applications. For example, Zhao et al. reported the synthesis of biodegradable amphiphilic PEO-b-PCL-grafted chitooligosaccharide (COS) copolymers and the corresponding supramolecular hydrogels with a-CD in aqueous solution [63]. Rheological studies of the asobtained hydrogels indicated that the physical properties could be modulated by controlling the concentration and graft content of the graft copolymers as well as the molar feed ratio of the graft to a-CD. In vitro release kinetics studies using BSA showed that the drug release profiles of this supramolecular hydrogel was strongly dependent on hydrogel composition.

Supramolecular hydrogels with dual temperature and $\mathrm{pH}$ responsiveness have been constructed by inclusion of PEO-grafted poly(dimethylacrylamide) with $a-C D$ in aqueous solution [64]. The temperature- or $\mathrm{pH}$-induced sol-gel transition of the system was found to be completely reversible, and it was shown that the chain uniformity, graft density and copolymer concentration can affect hydrogel behavior. The in vitro release of a model drug from this hydrogel was also found to be greatly accelerated at higher temperature and lower $\mathrm{pH}$, indicating potential applications in controlled drug delivery.

Matthews et al. recently demonstrated supramolecular hydrogel formation using $\alpha-C D$ and PEO-grafted poly ( $\alpha, \beta$-malic acid) [65]. In in vitro tests using doxorubicin hydrochloride, burst release of the drug was found to be minimized, and the release could be sustained for more than three days with associated suppression of cell viability due to effective application of anti-tumor activity.

\section{Conclusions}

Recent progress in the design of supramolecular inclusion complexes between cyclodextrin and various copolymers has inspired interesting developments in supramolecular biomaterials, particularly supramolecular hydrogels for drug delivery. Supramolecular hydrogels based on the self-assembly of inclusion complexes between CDs with biodegradable block copolymers could be used as a basis for injectable drug delivery systems that provide the sustained and controlled release of macromolecular drugs. The development of supramolecular systems has therefore had a major impact on research into drug delivery, opening up new possibilities for the design of novel drug delivery systems. When properly designed, the supramolecular structures can be controlled using many different copolymers, with a corresponding variety of drug delivery properties with a wide range of applications.

\section{References}

[1] J.-M. Lehn, Supramolecular Chemistry - Concepts and Perspectives (VCH, Germany, 1995).

[2] J.-P. Sauvage, C. Dietrich-Buchecker, Molecular Catenanes, Rotaxanes and Knots (VCH, Germany, 1999).

[3] J. A. Semlyen, Cyclic Polymers (Kluwer Academic Publishers, USA, 2000). 
[4] S. A. Nepogodiev, J. F. Stoddart, Chem. Rev. 98, 1959 (1998).

[5] F. M. Raymo, J. F. Stoddart, Chem. Rev. 99, 1643 (1999).

[6] P. R. Ashton et al., J. Am. Chem. Soc. 118, 4931 (1996).

[7] K. S. Chichak et al., Science 304, 1308 (2004).

[8] R. Schmieder, G. Hübner, C. Seel, F. Vögtle, Angew. Chem. Int. Ed. 38, 3528 (1999).

[9] F. Vögtle, T. Dünnwald, T. Schmidt, Accounts Chem. Res. 29, 451 (1996).

[10] H. W. Gibson, M. C. Bheda, P. T. Engen, Prog. Polym. Sci. 19, 843 (1994).

[11] H. W. Gibson, H. Marand, Adv. Mater. 5, 11 (1993).

[12] M. L. Bender, M. Komiyama, Cyclodextrin Chemistry (Springer-Verlag, Germany, 1978).

[13] J. Szejtli, Cyclodextrins and their Inclusion Complexes (Akademiai Kiado, Hungary, 1982)

[14] J. Szejtli, Chem. Rev. 98, 1743 (1998).

[15] G. Wenz, B. H. Han, A. Müller, Chem. Rev. 106, 782 (2006).

[16] Y. Chen, Y. Liu, Chem. Soc. Rev. 39, 495 (2010).

[17] N. A. Peppas, Hydrogels in Medicine and Pharmacy, Vol. 1. (CRC Press, USA, 1987).

[18] K. Park, W. S. W. Shalaby, H. Park, Biodegradable Hydrogels for Drug Delivery (Technomic Publishing Company, Inc., UK, 1993).

[19] A. Kishida, Y. Ikada, in Polymeric Biomaterials, S. Dumitriu ed. (Marcel Dekker, USA, 2002).

[20] J. Li, in Biomaterials Engineering and Processing Series, Vol. 1, S. H. Teoh ed. (World Scientific, USA, 2004).

[21] H. Park, K. Park, Pharm. Res. 13, 1770 (1996).

[22] S. Kim, Y. Bae, T. Okano, Pharm. Res. 9, 283 (1992).

[23] J. Heller, Adv. Drug Deliver. Rev. 10, 163 (1993).

[24] K. R. Kamath, K. Park, Adv. Drug Deliver. Rev. 11, 59 (1993).

[25] N. B. Graham, in Hydrogels in Medicine and Pharmacy, Vol. 2, N. A. Peppas ed. (CRC Press, USA, 1987).

[26] J. M. Harris, Poly(ethylene glycol) Chemistry: Biotechnical and Biomedical Applications (Plenum Press, USA, 1992).

[27] A. Apicella et al., in Polymeric Drugs and Drug Administration, M. Ottenbrite ed. (American Chemical Society, USA, 1994).

[28] P. Alexandridis, T. A. Hatton, Colloid. Surface. A 96, 1 (1995).

[29] L. E. Bromberg, E. S. Ron, Adv. Drug Deliver. Rev. 31, 197 (1998).

[30] B. Jeong, Y. H. Bae, D. S. Lee, S. W. Kim, Nature 388, 860 (1997).
[31] S. J. de Jong et al., Macromolecules 33, 3680 (2000).

[32] W. A. Petka, J. L. Harden, K. P. McGrath, D. Wirtz, D. A. Tirrell, Science 281, 389 (1998).

[33] G. Chen, A. S. Hoffman, Nature 373, 49 (1995).

[34] C. Wang, R. J. Stewart, J. KopeČek, Nature 397, 417 (1999).

[35] J. Li, X. J. Loh, Adv. Drug Deliver. Rev. 60, 1000 (2008).

[36] J. Li, Adv. Polym. Sci. 222, 79 (2009).

[37] J. Li, A. Harada, M. Kamachi, Polym. J. 26, 1019 (1994).

[38] J. Li, X. P. Ni, K. W. Leong, J. Biomed. Mater. Res. A 65A, 196 (2003).

[39] J. Li et al., Biomaterials 27, 4132 (2006).

[40] J. Li, X. Li, Z. Zhou, X. Ni, K. W. Leong, Macromolecules 34, 7236 (2001).

[41] X. J. Loh, S. H. Goh, J. Li, Biomacromolecules 8, 585 (2007).

[42] X. P. Ni, A. Cheng, J. Li, J. Biomed. Mater. Res. A 88A, 1031 (2009).

[43] Z. Y. Hui et al., J. Appl. Polym. Sci. 116, 1894 (2010).

[44] J. Li, X. Li, X. Ni, K. W. Leong, Macromolecules 36, 2661 (2003).

[45] X. Li, J. Li, K. W. Leong, Macromolecules 36, 1209 (2003).

[46] X. Li, J. Li, K. W. Leong, Polymer 45, 6845 (2004).

[47] J. Li et al., Langmuir 21, 8681 (2005).

[48] A. Harada, J. Li, M. Kamachi, Nature 356, 325 (1992).

[49] H. Luo, J. Phys. Chem. B 114, 4739 (2010).

[50] K. L. Liu, J. L. Zhu, J. Li, Soft Matter 6, 2300 (2010).

[51] K. L. Liu, S. H. Goh, J. Li, Polymer 49, 732 (2008).

[52] K. L. Liu, S. H. Goh, J. Li, Macromolecules 41, 6027 (2008).

[53] Y. Chen, X.-H. Pang, C.-M. Dong, Adv. Funct. Mater. 20, 579 (2010).

[54] X. Li, J. Li, J. Biomed. Mater. Res. A 86A, 1055 (2008).

[55] D.-Q. Wu et al., Langmuir 24, 10306 (2008).

[56] Wang et al., Biomaterials 30, 4161 (2009).

[57] K. M. Huh et al., Macromolecules 34, 8657 (2001).

[58] K. M. Huh et al., Macromol. Biosci. 4, 92 (2004).

[59] T. Nakama, T. Ooya, N. Yui, Polym. J. 36, 338 (2004).

[60] H. S. Choi et al., Macromol. Biosci. 2, 298 (2002).

[61] Y.-K. Joung, T. Ooya, M. Yamaguchi, N. Yui, Adv. Mater. 19, 396 (2007).

[62] R. Kawabata, R. Katoono, M. Yamaguchi, N. Yui, Macromolecules 40, 1011 (2007).

[63] S. Zhao, J. Lee, W. Xu, Carbohydr. Res. 344, 2201 (2009).

[64] L. Ren et al., Macromol. Biosci. 9, 902 (2009).

[65] A. S. Mathews, W.-J. Cho, I. Kim, C.-S. Ha, J. Appl. Polym. Sci. 113, 1680 (2009).

\section{Author profile}

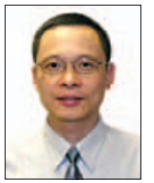

\section{Jun Li}

Jun Li received his BSc degree in chemistry from Sichuan University, China, in 1985, and his MSC and PhD degrees in macromolecular science from Osaka University, Japan, in 1992 and 1995. From 1995 he worked as a Special Postdoctoral Researcher at RIKEN in Japan, and in 1998 joined the A*STAR Institute of Materials Research and Engineering in Singapore, working concurrently with the National University of Singapore from 2002. He was a visiting scientist at Johns Hopkins University in the USA in 1998. He is now associate professor of the National University of Singapore and guest professor of Sichuan University, Southern Medical University and Shantou University in China. His recent research focuses on functional supramolecular structures and novel block copolymers as biomaterials for potential drug and gene delivery as well as tissue-engineering applications. 